\title{
THE RENEWABLE ENERGY IN A LED STANDALONE STREETLIGHT
}

\author{
D. VITALI, F. GARBUGLIA, V. D'ALESSANDRO \& R. RICCI \\ Dipartimento di Ingegneria Industriale e Scienze Matematiche, Università Politecnica delle Marche, Italy.
}

\begin{abstract}
This work deals with the design of a standalone streetlight provided with a solar panel and a multiple vertical axis wind turbine (VAWT) along the structure. A prototype was built and is currently being tested in the Monte Dago campus of the Università Politecnica delle Marche. The ongoing focus of the project is to improve the overall efficiency and the manufacturing details for the industrialization. A battery bank allows delaying the energy delivering from the energy production, while a central process unit on board collects the data from every component in the equipment. This unit allows to monitor the day-by-day efficiency of the energy-lighting system, and to send the information wirelessly with the purpose of integrating into a smart grid-like management platform. The test site includes a meteorological mast, which can measure the weather conditions, such as wind speed and solar radiation. The wind turbines included in the streetlight have been studied from an aerodynamic point of view through an extensive experimental analysis in the wind tunnel. Moreover, the structural design of the wind rotors was carried out together with the security system including a mechanical brake, which prevents the damage of the components during high wind speed conditions. The control of the hybrid energy unit, designed to track the optimal performance, has been analyzed throughout the local wind conditions. Also, it is discussed the effectiveness of this streetlight concept in various climate situations.

Keywords: LED streetlight, micro-wind, vertical axis wind turbine, wind-solar hybrid.
\end{abstract}

\section{INTRODUCTION}

The renewable energy technology today is maybe the main tool our society has to replace the fossil-fuel-based energy systems. Wind and solar energies, in particular, are green and free sources, available nearly everywhere and every day, especially, if we combine them in a hybrid apparatus capable of merging their statistical availability. What is not properly everywhere is the electrical energy network; consider the developing countries, for example. The resulting lack of public illumination can often represent a significant obstacle to the progress of those areas. With this regard, distributed renewable energy can play an important role in providing electricity close to its consumption. In particular, it has been proposed to integrate wind and solar energy directly into a lamppost prototype, making the outdoor lighting more independent of the present electrical network, or even fully standalone where the minimal climate conditions are met.

There are a few existent models following this path, either concepts or real commercial ones, which mainly adopt a single wind rotor and a photovoltaic panel, both near the top of the pole, and high-efficiency LED lights (e.g. [1-3]). Such micro-generation units, as quasistandalone lighting systems, generally have still to face the issues of standardization and reliability. These problems contribute to raise uncertainty in the distributed energy field.

This work addresses the matter through the implementation of a test site to study hybrid micro-wind and solar systems and the analysis of a novel hybrid streetlight prototype, already presented in Ricci et al. $[4,5]$ and developed by an academic-industrial partnership within a project sponsored by the Italian Ministry of Economic Development.

Its distinctive feature is the multi-pole structure specifically designed to house a multiple vertical axis wind turbine (VAWT), of Savonius type. Although VAWTs are drag-based rotors, with lower aerodynamic efficiency than traditional three-bladed horizontal axis wind turbines (HAWT), they are particularly suitable for micro-wind applications for mainly three reasons: 
- VAWTs have a robust construction, e.g. they do not have the complication of yaw control: they can capture wind energy from any wind direction.

- They are relatively slow and silent turbines, especially Savonius ones.

- They fit well into slender structures, possibly a key feature for urban integration.

In the next sections, various aspects of the project will be discussed, such as the structure of the prototype, the control of the wind turbines and the energy behavior of the system in the long term.

\section{THE PROTOTYPE}

The streetlight prototype is installed in the Monte Dago Engineering campus in Ancona, where has been tested and is further being developed.

The prototype has the possibility of a variety of architectural shapes, and visual impacts. It is shown in Fig. 1 in a former version with transparent straight wind rotors and in the latter configuration with helical fiber glass wind rotors.

\subsection{Design aspects}

The steel structure of the streetlight is $9 \mathrm{~m}$ high, and it has been conceived as a 4-pole vertical frame, uniformly enlarged in order to include the wind turbine train and the related hardware. This concept avoids both bulges and power units separated from the main frame. The four poles

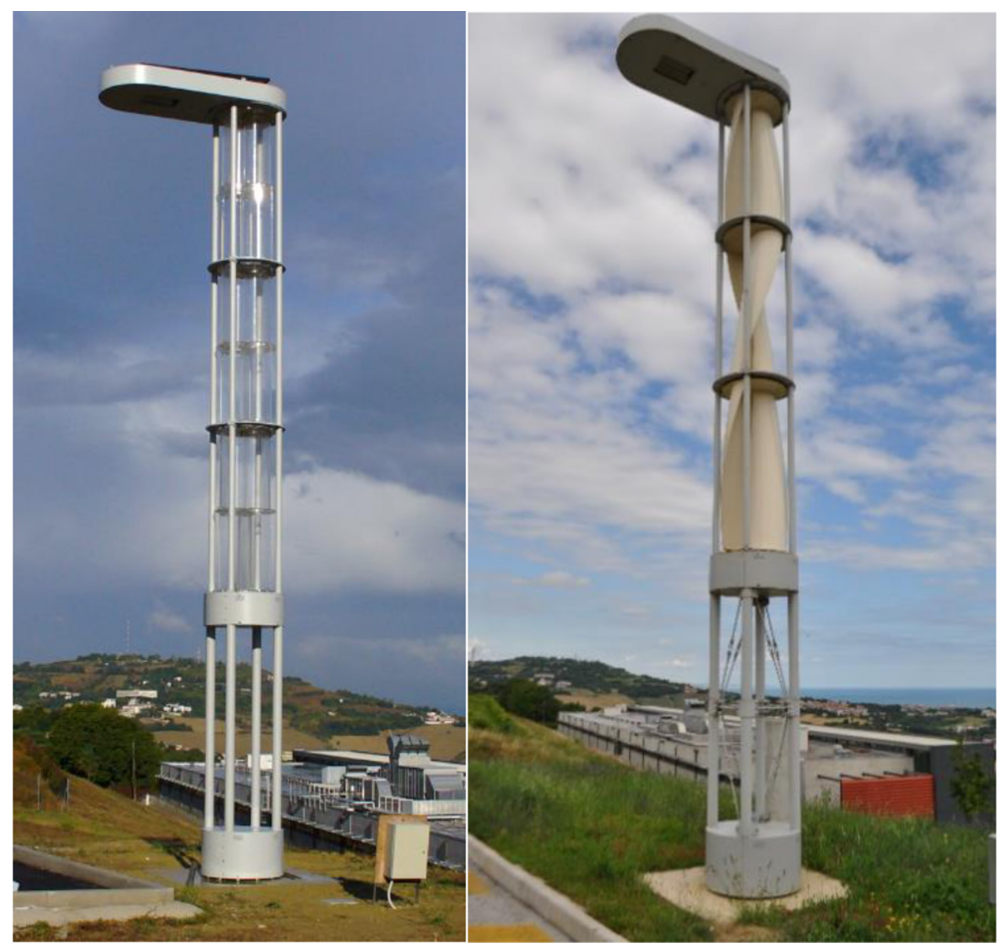

Figure 1: The streetlight prototype with the two different Savonius rotors: two-stage straight, transparent made, on the left; helical fiberglass made on the right. 
are bonded each other with several steel discs from base to top. They incorporate three coaxial Savonius wind modules, bonded through flanges and shafts to rotate jointly and to transmit the mechanical power to the electrical generator below, at a secure height of about $3 \mathrm{~m}$ (Fig. 2e).

The top casing houses the LED lamp, along with its current regulator, and it supports a nearly horizontal photovoltaic (PV) panel (Fig. 2a and b). This module contributes for the solar part of the hybrid system, which collects the electrical power in the electronic unit at the base of the lamppost (Fig. 2c), where a bank of four lead-acid batteries is located. Atop, there is also a cup anemometer (Fig. 2d) which helps keeping safe the system by monitoring the wind speed, both for the reference wind condition and for the detection of extreme conditions. The main specifications of the system are listed in Table 1.

The security of such a system is mostly related to the safe rotation of the wind turbines. Savonius rotor in particular lacks means of passive regulation, unlike HAWTs which can

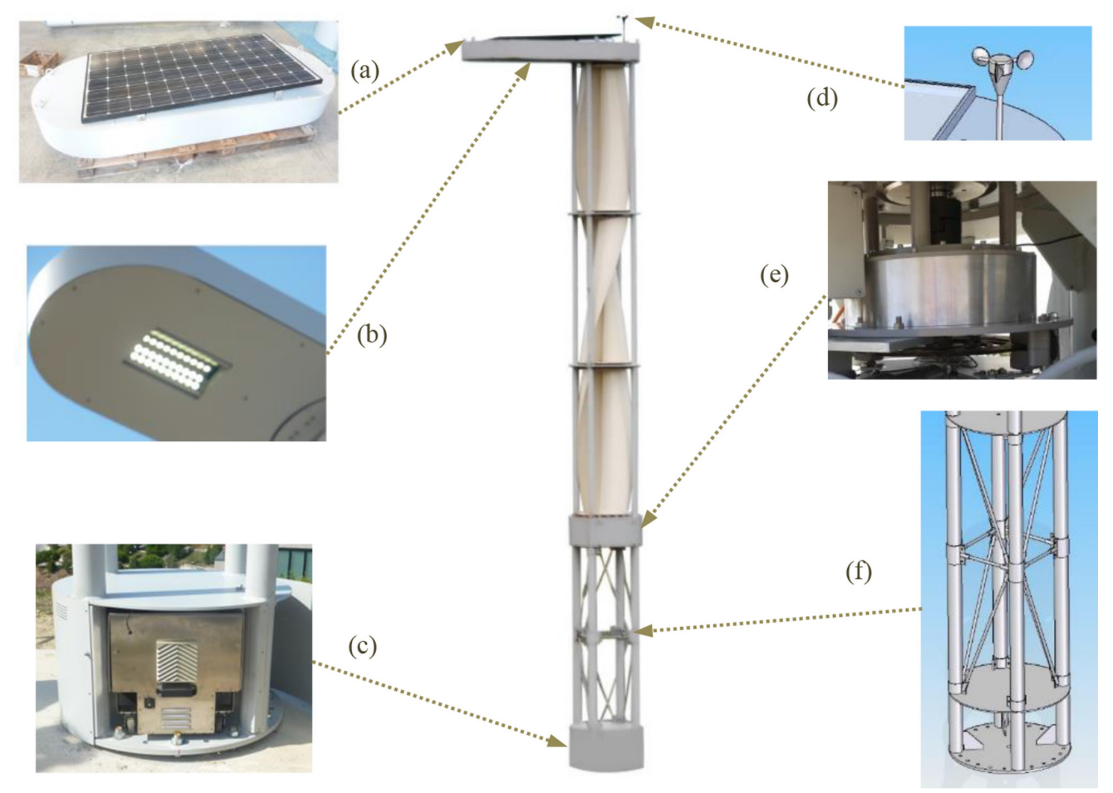

Figure 2: The prototype in the latter configuration. (a) PV panel; (b) LED lamp; (c) Power conversion and battery; (d) Control anemometer; (e) generator and brake; (f) base lattice structure.

Table 1: Specifications.

\begin{tabular}{ll}
\hline Size of the main structure & $0.9 \times 9 \mathrm{~m}$ \\
Wind rotor module & $0.7 \times 1.8 \mathrm{~m}$ \\
PV panel - rated power & $215 \mathrm{~W}$ \\
Lead acid battery bank & $40 \mathrm{Ah}$ at $48 \mathrm{~V}$ \\
Electrical generator & PMSG with 24 poles; direct drive \\
LED lamp & 6,000 lumens; 100 lumens/W \\
Wind Power conversion & 3-phase rectifier and controllable DC/DC converter \\
\hline
\end{tabular}


rely on the stall as a power-limiting phenomenon. When the rotation speed rises, the inertial loads, combined with the aerodynamic loads, happen to force the structure at increasing amplitudes and frequencies, eventually up to the resonance of the post. Such an over-speed could be dangerous both for the structure and for the people around. In fact, the deformation of the turbine material can even couple to the lamppost oscillations, and then induce collisions and damage to the material itself. The second bending natural frequency of the streetlight is quite in the range of an eventual over-speed, corresponding to $400 \mathrm{rpm}$ nearly. In order to contain the possible consequences, the initial support structure has been stiffened through lattices, as visible in Fig. 2f. This modification allows the above-mentioned bending frequency to rise to a much more secure level, reducing the risk of coupled oscillations.

The ordinary operation makes use of a redundant braking facility, triggered by either high wind speed or high $R P M$ value condition. The rotation is firstly slowed down by means of the alternator braking mode. Then, an automatic mechanical brake activates and stops the turbines completely. This system has been designed with the purpose of keeping the rotors safe during extreme wind speeds, without any steady energy consumption of the electric actuator. Figure 3 shows the kinematic scheme adopted to stop the brake disc.

\subsection{The Savonius wind turbines}

The Savonius rotors have been aerodynamically designed through a geometrical optimization performed in the wind tunnel of this University [6]. Two types resulted predominant from those tests: a straight-bucket and a $105^{\circ}$ helical-bucket Savonius, both with end-plates. Their aerodynamic performance, within a similar four-pillar structure, as the prototype has, was investigated in terms of the classical non-dimensional parameters for wind turbines [7]: the mechanical power coefficient $C_{p}$ and the tip speed ratio $\lambda$, defined in eqn (1).

$$
\begin{aligned}
\lambda & =\frac{\omega R}{U} \\
C_{P} & =\frac{P}{\frac{1}{2} \rho U^{3} \cdot A}
\end{aligned}
$$

The $105^{\circ}$ model presents a higher power coefficient than the straight one in this condition: its maximum wind tunnel-derived value is $C_{p}=0.21$ at $\lambda=0.94$.

The straight Savonius module was further developed into two staggered stages in order to smooth down the torque variability over the round and to increase the overall rotor stiffness
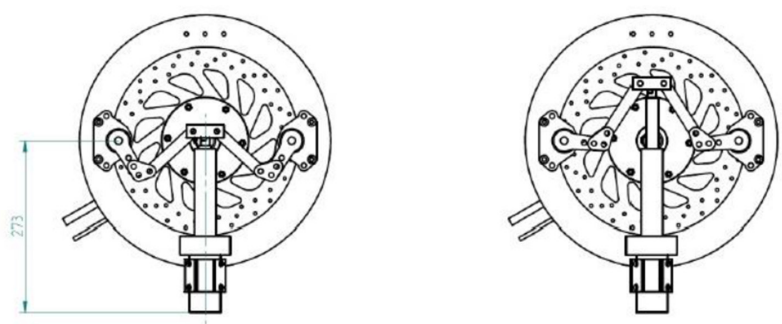

Figure 3: Disc brake mechanism: actuation kinematics. 

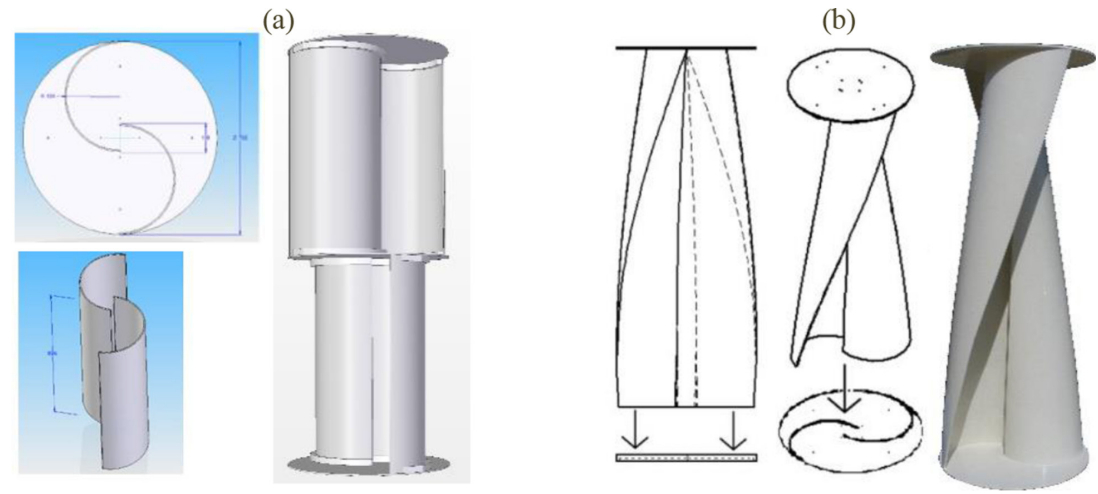

Figure 4: The Savonius wind rotors: straight two-stage on the left; $105^{\circ}$ helical on the right.

thanks to the intermediate plate (Fig. 4a). The material selected for this type was transparent acrylic glass (PMMA).

The helical Savonius module, currently installed, is made of glass-reinforced plastic (GRP) instead, specifically fiber glass in polyester. This material exhibits better mechanical properties than PMMA: not only can the shaft be excluded from the wind rotor but also the intermediate plate. A stress analysis was performed considering the centrifugal loads during over-speeds and extreme wind loads with no rotation. In order to operate in a fully secure stress level, buckets and plates were built with four layers of fiber fabric, which resulted into a final thickness of nearly $5 \mathrm{~mm}$ (Fig. 4b).

\section{THE TEST SITE AND ENERGY SIMULATIONS}

\subsection{The meteorological data}

A meteorological mast has been installed on the hill nearby the prototype (Fig. 5) in order to measure the climate variables over long periods and characterize the site from a wind energy point of view. The mast has been equipped with the following instrumentation.

- Cup anemometer for the wind speed, at $10 \mathrm{~m}$ (agl).

- Wind goniometer for the wind direction, at $10 \mathrm{~m}$ (agl).

- Barometer for the atmospheric pressure.

- Air temperature and humidity sensor.

- Solar radiometer for the global radiation.

- Solar radiometer for the direct radiation.

- Data logger with GPRS communication system: variables averaged over 10 min periods.

A 1-year data from the meteorological tower allowed to perform a statistical analysis of the available wind energy in this site. The wind frequency rose in Fig. 5 shows that the principal wind directions are from NNW (from the sea) and from south. With the latter direction, the wind turbines happen to be aerodynamically shadowed by the hill, slowing down the effective local wind speed.

The wind power density $D(u)$ has been evaluated using a Weibull probability density function $f(u)$ fitted over the annual wind occurrences. 

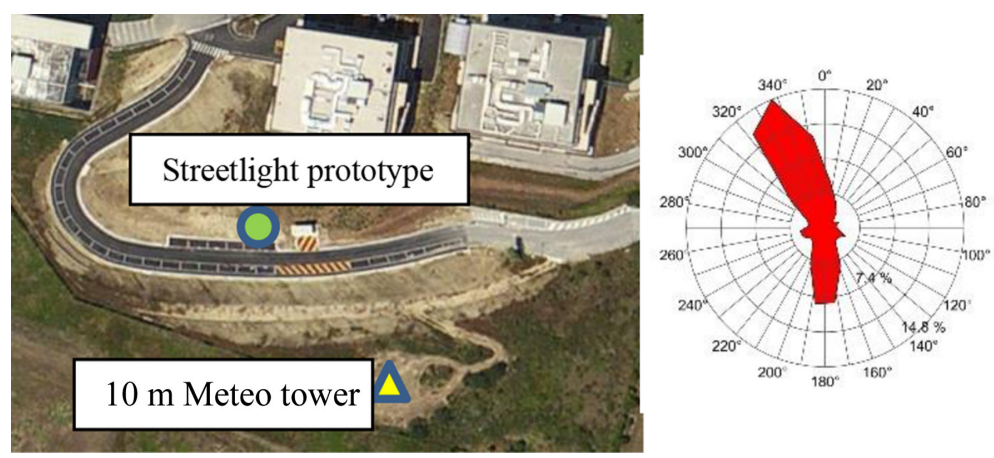

Figure 5: Map of the test site in the Monte Dago Engineering University campus and oneyear wind frequency rose.

$$
D(u)=f(u) \frac{1}{2} \rho u^{3}
$$

This formula estimates the average available wind power per square meter, in the long term. In the Monte Dago campus, the average measured wind speed is $3.7 \mathrm{~m} / \mathrm{s}$, while the maximum power density results to be nearly $11 \mathrm{~W} / \mathrm{m}^{2}$, at $u=6.0 \mathrm{~m} / \mathrm{s}$. This available power has to be exploited by the turbine frontal area, according to its effective power coefficient $C_{p}$.

The air density was also derived from the pressure and temperature: the mean value was $\rho=1.20 \mathrm{~kg} / \mathrm{m}^{3}$ with a standard deviation of 0.03 over the year.

\subsection{The energy simulation}

In order to predict numerically the effectiveness of this concept of streetlight in the long term, an energy simulation with MATLAB has been performed. The different components of the hybrid energy system have been modeled mainly as efficiency curves and subjected to 10 min-averaged weather data from the meteorological mast. A particular attention has been paid to the logical states of the controller, e.g. for the battery the low charge condition prevents the lamp from switching on. In Table 2 the main assumptions of the model are summarized.

Moreover, a function has been implemented to estimate the effect of a possibly different siting for the prototype. The available renewable energy, specific for the site, can indeed affect deeply the autonomy of such a system from any type of energy backup. This function alters the wind speed and the global radiation signals fed to the model. While the wind speed is simply scaled, the effect of different latitude is taken into account with the following approximated criteria:

- the radiation is spread according to the local duration of the day.

- the scale factor applied to the radiation signal is based on the daily solar energy ratios in clear sky condition, between the two latitudes: that is related to the different sun elevation.

- the evolution of cloudiness remains the same as measured in Monte Dago hill (nearly $43^{\circ}$ of latitude, for instance). 
Table 2: Energy model hypothesis.

\begin{tabular}{|c|c|}
\hline Electrical generator & $\begin{array}{l}\text { Analytical efficiency fitting surface } \\
\eta_{e l}(\text { torque, } r p m)\end{array}$ \\
\hline Wind turbines & $\begin{array}{l}105^{\circ} \text { helical modules with quasi-steady power curve; vari- } \\
\text { able speed until } 200 \mathrm{rpm}\end{array}$ \\
\hline Wind speed & $\begin{array}{l}\text { Scaled to the prototype with experimental speed-up factors; } \\
\text { vertical wind shear typical for fallow fields }\end{array}$ \\
\hline Battery & $\begin{array}{l}\text { With internal resistance and coulombic efficiency of } 0.8 \text {; } \\
\text { steady capacity over time }\end{array}$ \\
\hline PV panel & $\begin{array}{l}\text { Steady overall efficiency of } 16 \% \text {; the global radiation is the } \\
\text { source }\end{array}$ \\
\hline Electrical load & $\begin{array}{l}\text { LED switching according to the global radiation as night } \\
\text { detector; continuous electronics consumption of } 15 \mathrm{~W}\end{array}$ \\
\hline Power DC/DC conversion & Electrical efficiency of $90 \%$ \\
\hline
\end{tabular}

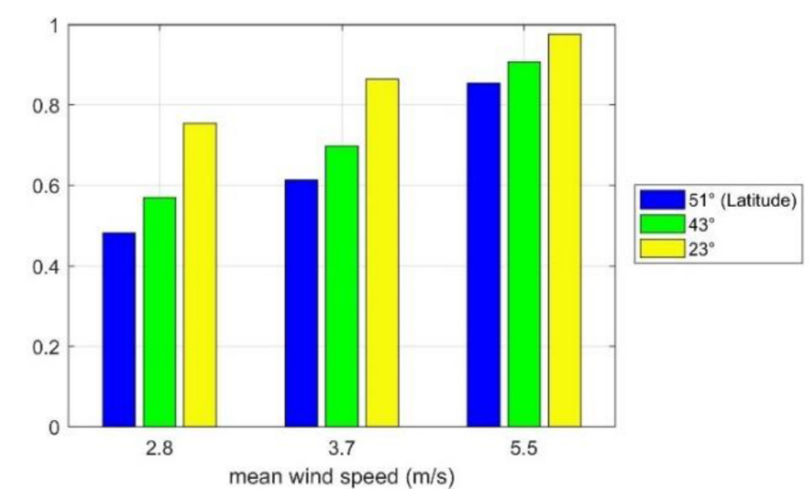

Figure 6: Annual portion of met lighting time for various simulated scenarios (different wind and solar conditions).

The most important parameter that results from the energy simulations is the annual ratio of met lighting time. The total number of hours a streetlight is required to be on, depends naturally on the local and current duration of the day. The simulations have been performed considering the system in standalone mode and have pointed out that in these conditions the simulated prototype can satisfy the lighting nearly in the $70 \%$ of the required hours. In Fig. 6 , there is a comparison of the relative met lighting time in different renewable sources combinations. The solar radiation in various latitudes affects both the available photovoltaic energy and the electrical load demand, in terms of night durations, particularly in winter.

\section{THE CONTROL}

The design of the control for such a hybrid renewable energy system is composed of two main purposes: the optimal energy production and storage, and the safety of components. A remarkable aspect that connects these issues is for example the maximum charging current allowed to flow safely into the battery. In certain circumstances, this current threshold can limit the resistant torque applied to the wind turbine, eventually giving sudden over-speeds. 
In order to manage this issue, a dump load was adopted to dissipate the possible over-current into external resistors.

The Maximum Power Point Tracking (MPPT) for the photovoltaic generator was implemented as a constant voltage algorithm in the solar DC/DC converter. The control of the wind turbines, on the other hand, has been designed as an optimal generator torque controller, as there are no movable aerodynamic surfaces in the adopted wind rotors.

\subsection{The rotation control}

In the micro-wind sector, the variable speed operation is both feasible, by using an electronically controlled PMSG alternator, and desirable to enhance the start-up and better exploit a typically poor wind resource, for this application. Within the maximum rotation limit, due to structural safety, the generator torque should increase quadratically with rotation speed as $(R P M)^{2}[8]$, so that the turbine can track the optimum point in the $C_{p}-\lambda$ curve and then maximize the aerodynamic efficiency, at least in quasi-steady conditions. The ideal torque curve for the wind rotors installed on the prototype is represented in Fig. 7 as circle markers. The rotation maximum limit here is $200 R P M$.

The size of the circles is proportional to the power density $D(u)$ (eqn (2)) of the local wind, which is indicated by the grey lines. This hints the reason why the cut-off wind speed has been fixed at $14 \mathrm{~m} / \mathrm{s}$ only.

The isolines of generator efficiency have been calculated fitting the curves present in the related data-sheet. Such electrical efficiency shows some drawbacks in the lower working range. Indeed, the optimum tip speed ratio $\left(\lambda_{\text {opt }}\right)$ actually depends also on the efficiency curve of the alternator. This electrical efficiency turns to increase with the rotation speed, giving a

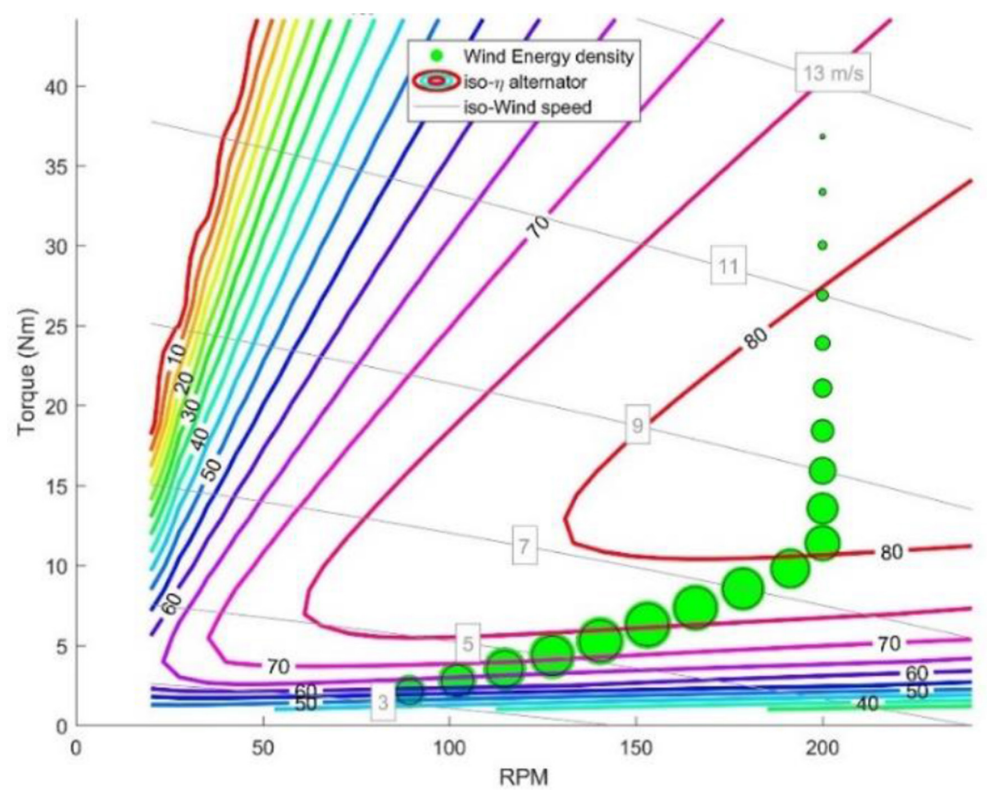

Figure 7: Ideal generator torque curve (circle markers), over iso-curves of wind speed and generator electrical efficiency. The circle size is proportional to $D(u)$. 
combined $\lambda_{o p t}$ slightly larger than the pure aerodynamic value: it goes approximately from 0.94 to 0.96 .

The actual rotation control has been implemented using the generator voltage as control variable.

\subsection{Effects of the wind profile}

A multi-VAWT like in the present prototype happens to have a relatively high vertical extension. This makes the vertical wind shear important for an effective wind source estimation. Here, a logarithmic profile [9] is applied to the involved heights in order to evaluate its effects on the turbines individually (Fig. 8).

$$
u(z)=\frac{u^{*}}{k} \ln \left(\frac{z}{z_{0}}\right)
$$

This formula gives the logarithmic factors for the downscaling of the average wind speed, from the top anemometer height down to the three Savonius modules. The key parameter here is $z_{0}$, which is the surface roughness length and ultimately describes the level of asperities present in the surrounding ground terrain.

The value of $z_{0}$ increases as the terrain gets rougher: the feasible range goes from the scale of millimetres, over the sea or nearby, where the wind profile is more uniform, to the scale of one meter in the centres of cities.

The non-uniform wind speed also introduces a dissimilarity in the individual $\lambda$ for each rotor. In particular, only the central module, the one at $6 \mathrm{~m}$ agl, will almost work at the optimal rotation and the maximum efficiency $C_{P}$. If you consider the top anemometer signal as the reference wind speed for the whole multi-VAWT, the overall effective $\lambda$ will be reduced with respect to the optimal $\lambda$ values derived from the wind tunnel tests. In Fig. 9 (dashed curve), it is shown how $\lambda$ should change with wind speed according to the power curve of Fig. 7. In the variable speed zone, approximately in the range $4-9 \mathrm{~m} / \mathrm{s}$, the different wind profile affects significantly the total tip speed ratio $\lambda$.

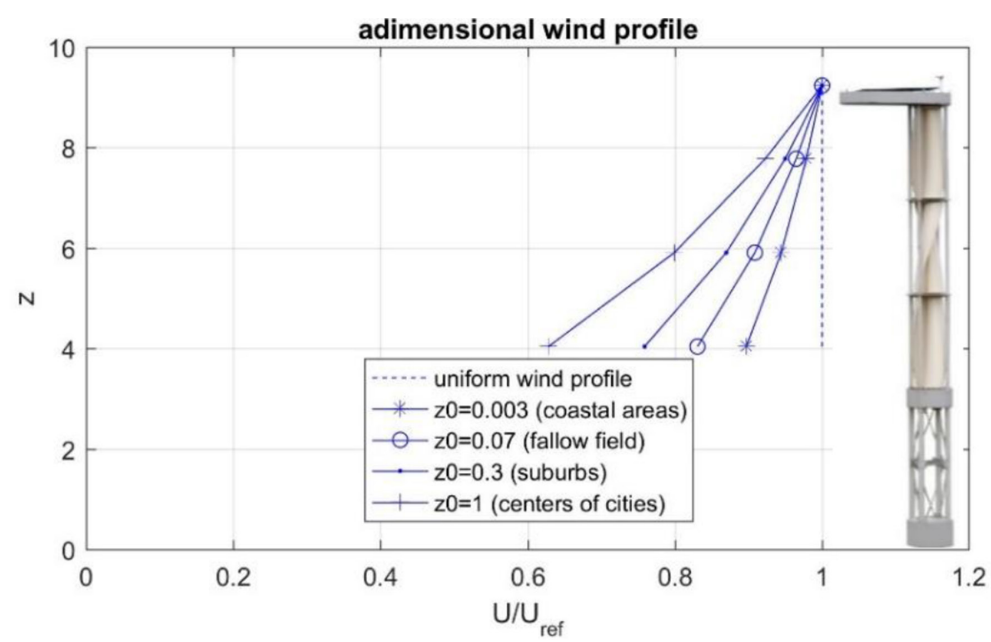

Figure 8: Non-dimensional wind profiles at different values of $z_{0}$, over the prototype. 


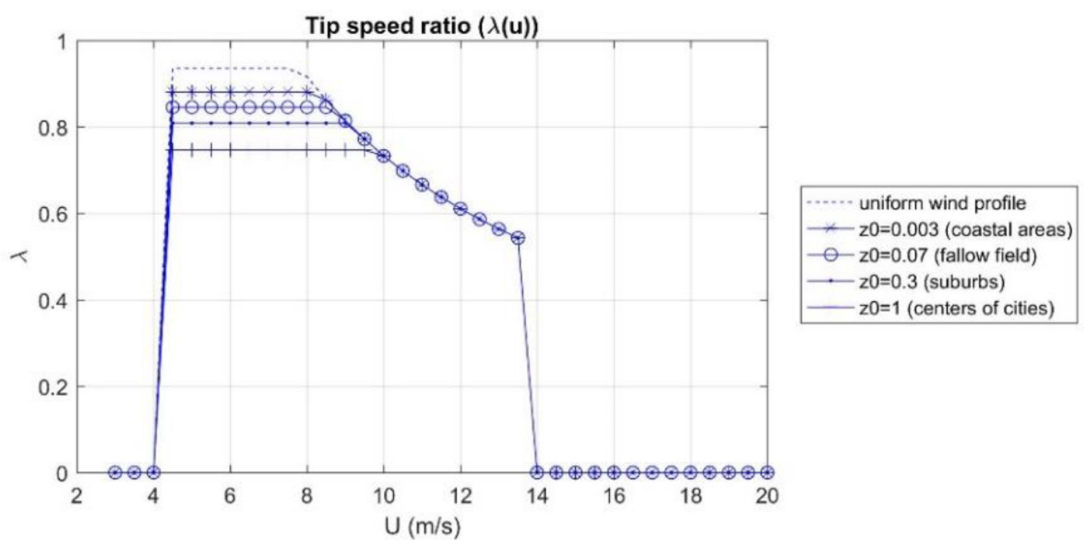

Figure 9: Overall tip speed ratio against wind speed curves, with different $z_{0}$ values.

Such an off-design tip speed ratio happens to cut down the overall aerodynamic efficiency of the multiple turbines. According to this estimation, the maximum attainable $C_{P}$ value can fall from 0.21 down to 0.15 in the case of 'fallow field' terrain, or even to 0.10 in a wind shear typical for city centres.

\section{REMARKS ON THE OPERATING DATA}

The experimental data collected during the tests were the wind speed from the top anemometer, the rounds per minute of the rotors and all the electrical magnitudes involved in the power conversion such as the current charging the battery and the rectified DC voltage at the output of the generator. The wind power too has been evaluated, starting from the voltage and current measures on the output of the dedicated DC/DC converter. A one-month instantaneous data set, for the configuration with the two-stage straight Savonius models, are reported in Fig. 10, against the top anemometer wind speed. The significant scattering of the points

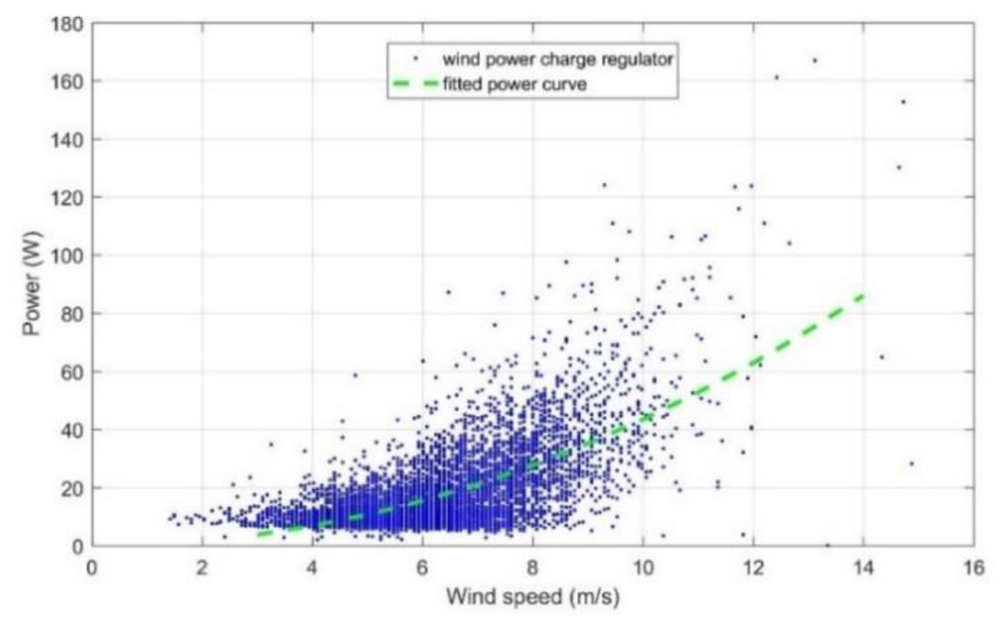

Figure 10: Electrical power from the wind system to the batteries, against the top anemometer wind speed (1-month data). 
with respect to the fitting power curve is caused mainly by the transient wind conditions. Also, the direction of the wind can play a role as the wind profile, along with $z_{0}$, depends on the various obstacles around the prototype. The average power curve from the Savonius wind turbines is represented with the dashed line. These values are the outcome of the aerodynamic power coefficient combined with the drive train and the electronic converter efficiencies. In the current site, the overall power coefficient results to reach a value of $6 \%$, while the rotation control, implemented so far, does give priority to the security against over-speeds, rather than to the optimal $\lambda$ tracking. Indeed, the turbines decrease their operating $\lambda$ gradually with increasing wind speed, down to a value of nearly 0.3 at the cut-out wind. The local wind conditions, included turbulence and wind shear, ultimately happen to affect the control parameters of the prototype. Hence, the biggest challenge for the further development of this hybrid energy streetlight is to make the wind control system more versatile, implementing an adaptive parametric algorithm for the variety of local situations. The site-specific optimal $\lambda$ is indeed recommended to be automatically detected using the feedback of the effective generated power.

\section{REFERENCES}

[1] UGE Outdoor Lighting, available at: http://www.ugei.com/products/lighting.

[2] Eco Smart inc, available at: http://www.ecosmartinc.com/catlite26.php.

[3] Berdanier, R.A., Hernandez, K.E., Raye, C.P., Horvath, C.P., Graham, L.M., Hatlee, T.P., Phan, N.H., Pelken, P.M. \& Dang, T.Q., Integrating vertical-axis wind turbines and photovoltaic solar cells to power a self-sustaining outdoor light source. In 7th International Conference on Indoor Air Quality, Ventilation and Energy Conservation in Buildings, Syracuse, New York, 2010.

[4] Ricci, R., Vitali, D. \& Rossi, M., An innovative wind/solar hybrid street lighting system: development and early testing of a prototype. In MicrogenIII: Proceedings of The 3rd edition of the International Conference on Microgeneration and Related Technologies, Naples and Italy, April 15-17, 2013.

[5] Ricci, R., Vitali, D. \& Montelpare, S., An innovative wind-solar hybrid street light: development and early testing of a prototype. International Journal of Low-Carbon Technologies, 10(4), pp. 420-429, 2015. http://dx.doi.org/10.1093/ijlct/ctu016

[6] Ricci, R., Romagnoli, R., Montelpare, S. \& Vitali, D., Experimental study on a Savonius wind rotor for street lighting systems. Applied Energy, 161, pp. 143-152, 2016. http://dx.doi.org/10.1016/j.apenergy.2015.10.012

[7] Burton, T., Wind Energy: Handbook, John Wiley \& Sons: Chichester and New York, 2001.

[8] Hansen, M.O.L., Aerodynamics of Wind Turbines, Earthscan: London and Sterling, VA, 2008.

[9] Manwell, J.F., McGowan, J.G. \& Rogers, A.L., Wind Energy Explained: Theory, Design and Application, John Wiley \& Sons: Chichester, U.K, 2009. 